\title{
Less burn, more fat: electronic cigarettes and pulmonary lipid homeostasis
}

\author{
Aran Singanayagam and Robert J. Snelgrove \\ Inflammation Repair and Development, National Heart and Lung Institute, Imperial College London, London, United Kingdom.
}

\begin{abstract}
Electronic nicotine delivery systems (ENDS) are rapidly increasing in popularity due to the perception that they may represent a safe alternative to conventional cigarettes. However, a growing body of evidence indicates that ENDS exposure can disrupt maintenance of pulmonary immune homeostasis and antimicrobial immunity. In this issue of the $J C l$, Madison et al. demonstrate that in mice, chronic ENDS exposure induces profound alterations in lipid homeostasis. ENDS-exposed mice showed irregularities in the surfactant-secreting lamellar bodies within type 2 alveolar cells and increased intracellular phospholipid accumulation within alveolar macrophages. Moreover, ENDS-exposed mice displayed greater inflammation and tissue damage in response to influenza $A$, which may be due to downregulated expression of a viral pattern-recognition receptor in alveolar macrophages. Collectively, the results of this study identify previously unrecognized adverse effects of ENDS exposure on pulmonary lipid metabolism, although the implication of these effects on long-term respiratory health requires future exploration.
\end{abstract}

\section{Electronic nicotine delivery} systems - what's the harm?

The adverse health consequences of longterm cigarette smoking are well established, and it remains the leading cause of preventable death worldwide (1). Although tobacco use is steadily declining, there has been a rapid increase in the popularity of electronic nicotine delivery systems (ENDS, also known as e-cigarettes), particularly among adolescents (2). This is partly due to the perception that ENDS may offer a safer alternative to conventional cigarettes and a potentially useful means to facilitate smoking cessation (3), although many of the adolescent users were previously non-smokers. ENDS deliver a liquid consisting of variable concentrations of nicotine within the solvents propylene glycol (PG) and vegetable glycerine (VG), which is then heated to produce a vapor that can be inhaled directly into the lungs. There are additionally more than 7,500 unique flavors created by the inclusion of various additives (4).

Numerous regulatory mechanisms act in concert to maintain pulmonary homeostasis, and this delicate balance can be disrupted by inhalation of toxins. Despite containing fewer potentially harmful chemicals than tobacco smoke, emerging evidence indicates that inhalation of ENDS vapor can disrupt a wide range of processes involved in maintenance of pulmonary immune homeostasis. In vitro exposure to components of ENDS liquids/ vapors induces proinflammatory effects in bronchial epithelial cells $(5,6)$, alveolar macrophages (7), and neutrophils (8). Impaired host-defense functions including reduced macrophage phagocytosis (9, 10), accentuated bacterial adherence (11),

Delated Article: p. 4290

Conflict of interest: The authors have declared that no conflict of interest exists.

and altered neutrophil extracellular trap (NET) formation (9) have also been reported. As it is difficult to accurately recapitulate the complexities of cellular exposure to inhaled vapor using an in vitro system, other studies have sought to use animal models to further understand the immunological effects of ENDS. These studies have reported airway inflammation (acute transient [ref. 12] or chronic [ref. 5]), reduced lung function (13), and impaired mucociliary function $(5,14)$, with some (5), but not all (15), studies also reporting emphysematous lung changes. Differences in exposure delivery systems and vapor used (variable addition of nicotine or flavorings) may explain the contrasting findings observed in some of these studies, although they uniformly demonstrate that ENDS exposure affects several aspects of pulmonary immune homeostasis. There are very few human in vivo studies to date, but ENDS users have been shown to have elevated airway matrix metalloproteinase 9 and NET-related proteins (16) and increased expression of the mucin glycoprotein MUC5AC $(16,17)$.

\section{Dysregulated lipid metabolism and disease}

Maintenance of lipid metabolism is crucial for cellular function in all tissues, and perturbations in lipid homeostasis occur in a range of human diseases. Pulmonary surfactant, which maintains alveolar surface tension, is approximately $90 \%$ lipid (18). There is a growing body of evidence indicating that pulmonary surfactant and associated lipid metabolism has broader roles in maintenance of pulmonary immune homeostasis and host defense. Thus, any exposure that interferes with these critical processes may be expected to have profound effects on the lung microenvironment.

In this issue, Madison et al. employed a mouse model of chronic inhalational exposure to study the in vivo effects of ENDS-associated vehicle solvents PG and VG in comparison to conventional tobac- 
co smoke exposure (19). In contrast with tobacco smoke, the authors observed no emphysematous changes nor increased airway inflammation following 4 months of exposure to ENDS (with or without nicotine). Despite not showing any measurable effects on inflammation or lung architecture, Madison et al. identified profound alterations in pulmonary lipid homeostasis associated with ENDS vapor exposure. Alveolar macrophages taken from ENDS-exposed animals displayed increased lipid aggregates within the cytoplasm, independent of the presence of nicotine. Abnormalities were also observed in the lamellar bodies (surfactant-secreting organelles) within type 2 alveolar cells of ENDS-exposed mice. Interestingly, the authors did not observe similar effects in macrophages from tobacco smokeexposed mice, suggesting that this could be an effect unique to ENDS solvents, although a previous study suggested that a longer duration (6 months) of tobacco smoke exposure in mice can promote lipid accumulation in macrophages (20). Using mass spectrometry, the authors further characterized the specific alterations induced by ENDS exposure, demonstrating an increase in cellular phospholipid species, which was confirmed by measurement of intracellular phospholipids in cells isolated by bronchoalveolar lavage (BAL) (19). Furthermore, ENDS exposure upregulated expression of Abca3, which encodes a type II cell-specific lipid transport protein critical for lamellar body biogenesis (21) and Pcyt1a and Lpcat1, which encode phosphatidylcholine-synthesizing enzymes.

In addition to increasing lipid species, ENDS exposure also altered the protein component of surfactant by reducing the concentration of BAL surfactant protein D (SP-D) (19). Intriguingly, the phenotype observed in ENDS-exposed mice was strikingly similar to that reported previously in mice with deletion of SP-D (22), which display increased surfactant lipids in lung tissue and airways associated with increased numbers of foamy macrophages. Thus, it is possible that the reduction in SP-D resulting from ENDS exposure is a central driver in subsequent alterations in lipid homeostasis. Further studies to evaluate the ability of exogenous SP-D administration to restore lipid homeostasis in ENDSexposed mice will be informative.

\section{Pulmonary lipid dysregulation and antiviral host defense}

Madison et al. also showed that ENDS exposure in mice was associated with downregulated M1-associated macrophage markers including $T l r 7$, a patternrecognition receptor (PRR) that senses viral single-stranded RNA (19). This prompted further investigation into the effects of ENDS in a mouse infection model of the common respiratory virus influenza A. Compared with air-treated controls, ENDS-treated mice challenged with influenza had augmented airway inflammation, evidence of lung hemorrhage/ edema, and greater weight loss, suggestive of increased virus-induced pathology. Whether these effects were driven by an impaired innate antiviral response secondary to downregulation of viral PRRs or an alternative mechanism was not explored, and represents an area of future interest. Furthermore, it is unclear at present whether the adverse response to influenza challenge observed in ENDS-treated mice is a direct consequence of the aberrant lipid homeostasis, or an independent manifestation of ENDS use. A previous study revealed that ENDS liquid impairs in vitro induction of the epithelial host-defense peptide SPLUNC1 in response to rhinovirus infection (23), and thus numerous protective antiviral responses could potentially be impaired by ENDS use. Interestingly, SP-D has previously been shown to play a role in host defense against influenza virus infection in mice (24), suggesting that reduction in this protein could be central to the deleterious effects of ENDS.

\section{Future directions}

What are the implications of these findings for the ever-growing number of ENDS users? Clearly, these potentially adverse effects on lipid homeostasis with associated impairment of innate immune function could translate to greater frequency and/ or severity of respiratory tract infections in individuals who persistently use these devices. The long-term consequences of such episodes for respiratory health, particularly related to lung development in younger individuals, is unclear. Notably, a previous study reported that ENDS use is associated with increased rates of chronic bronchitic symptoms in adolescents (25). The long-term consequences of the pro- found changes in lipid metabolism associated with ENDS exposure are also unclear. Altered lipid homeostasis has been reported in chronic obstructive pulmonary disease (COPD), characterized by increased airway expression of sphingolipids (26), although it remains to be determined whether altered lipid homeostasis is causally related to chronic lung disease. However, use of ENDS as smoking cessation devices in COPD patients may potentially expose patients with preexisting altered lipid homeostasis to more profound perturbations. Additionally, as COPD is associated with increased propensity for virus-induced exacerbations, impairment of antiviral immunity by ENDS use could further accentuate the frequency and/or severity of exacerbations in these already susceptible patients.

As it is difficult to recapitulate the complexities of vapor inhalation in a smallanimal exposure system, human vaporchallenge studies will be required to confirm that the effects observed in mouse models also occur in humans. Whether alternative solvents to PG and VG that exert lesser effects on immune homeostasis could be employed requires future consideration. Furthermore, the ENDS used in the study by Madison et al. (19) are only used by less than $10 \%$ of vapers. Alternative flavorings and different delivery devices add a further layer of complexity that may affect these altered processes, warranting future investigation. These important studies add to the growing consensus that promotion of ENDS as safe alternatives to tobacco should be viewed with caution, and have important implications for the manufacturing, marketing, and consumption of ENDS.

\section{Acknowledgments}

AS is supported by a Wellcome Trust Imperial College Institutional Strategic Support Springboard Fellowship. RJS $(209458 / \mathrm{Z} / 17 / \mathrm{Z})$ is a Wellcome Trustfunded Senior Research Fellow in Basic Biomedical Sciences.

Address correspondence to: Robert J. Snelgrove, National Heart and Lung Institute, Imperial College London, London SW7 2AZ, United Kingdom. Phone: 44.0. 207.594.8192; Email: robert.snelgrove@ imperial.ac.uk. 
1. WHO. WHO Global Report on Mortality Attributable to Tobacco. Geneva, Switzerland: WHO Tobacco Free Initiative; 2012.

2. WHO. Electronic nicotine delivery systems and electronic non-nicotine delivery systems. Paper presented at: Conference of the Parties to the WHO Framework Convention on Tobacco Control; November 7-12, 2016; Delhi, India. https:// www.who.int/fctc/cop/cop7/FCTC_COP_7_11_ EN.pdf. Accessed August 16, 2019.

3. Amrock SM, Zakhar J, Zhou S, Weitzman M. Perception of e-cigarette harm and its correlation with use among U.S. adolescents. Nicotine Tob Res. 2015;17(3):330-336.

4. Kaisar MA, Prasad S, Liles T, Cucullo L. A decade of e-cigarettes: Limited research \& unresolved safety concerns. Toxicology. 2016;365:67-75.

5. Garcia-Arcos I, et al. Chronic electronic cigarette exposure in mice induces features of COPD in a nicotine-dependent manner. Thorax. 2016;71(12):1119-1129.

6. Higham A, Bostock D, Booth G, Dungwa JV, Singh D. The effect of electronic cigarette and tobacco smoke exposure on COPD bronchial epithelial cell inflammatory responses. Int $J$ Chron Obstruct Pulmon Dis. 2018;13:989-1000.

7. Scott A, et al. Pro-inflammatory effects of e-cigarette vapour condensate on human alveolar macrophages. Thorax. 2018;73(12):1161-1169.

8. Higham A, et al. Electronic cigarette exposure triggers neutrophil inflammatory responses. Respir Res. 2016;17(1):56.

9. Clapp PW, et al. Flavored e-cigarette liquids and cinnamaldehyde impair respiratory innate immune cell function. Am J Physiol Lung Cell Mol
Physiol. 2017;313(2):L278-L292.

10. Ween MP, Whittall JJ, Hamon R, Reynolds PN, Hodge SJ. Phagocytosis and inflammation: Exploring the effects of the components of e-cigarette vapor on macrophages. Physiol Rep. 2017;5(16):e13370.

11. Miyashita L, et al. E-cigarette vapour enhances pneumococcal adherence to airway epithelial cells. Eur Respir J. 2018;51(2):1701592.

12. Glynos C, et al. Comparison of the effects of e-cigarette vapor with cigarette smoke on lung function and inflammation in mice. Am JPhysiol Lung Cell Mol Physiol. 2018;315(5):L662-L672.

13. Larcombe AN, Janka MA, Mullins BJ, Berry LJ, Bredin A, Franklin PJ. The effects of electronic cigarette aerosol exposure on inflammation and lung function in mice. Am J Physiol Lung Cell Mol Physiol. 2017;313(1):L67-L79.

14. Chung S, et al. Electronic cigarette vapor with nicotine causes airway mucociliary dysfunction preferentially via TRPA1 receptors [published online ahead of print June 7, 2019]. Am J Respir Crit Care Med. https://doi.org/10.1164/ rccm.201811-2087OC.

15. Crotty Alexander LE, et al. Chronic inhalation of e-cigarette vapor containing nicotine disrupts airway barrier function and induces systemic inflammation and multiorgan fibrosis in mice. Am J Physiol Regul Integr Comp Physiol. 2018;314(6):R834-R847.

16. Reidel B, et al. E-cigarette use causes a unique innate immune response in the lung, involving increased neutrophilic activation and altered mucin secretion. Am J Respir Crit Care Med. 2018;197(4):492-501.
17. Ghosh A, et al. Chronic e-cigarette exposure alters the human bronchial epithelial proteome. Am J Respir Crit Care Med. 2018;198(1):67-76.

18. Han S, Mallampalli RK. The role of surfactant in lung disease and host defense against pulmonary infections. Ann Am Thorac Soc. 2015;12(5):765-774.

19. Madison MC, et al. Electronic cigarettes disrupt lung lipid homeostasis and innate immunity independent of nicotine. JClin Invest. 2019;129(10):4290-4304.

20. Morissette MC, Shen P, Thayaparan D, Stämpfli MR. Disruption of pulmonary lipid homeostasis drives cigarette smoke-induced lung inflammation in mice. Eur Respir J. 2015;46(5):1451-1460.

21. Cheong N, et al. ABCA3 is critical for lamellar body biogenesis in vivo. J Biol Chem. 2007;282(33):23811-23817.

22. Korfhagen TR, et al. Surfactant protein-D regulates surfactant phospholipid homeostasis in vivo. J Biol Chem. 1998;273(43):28438-28443.

23. Wu Q, Jiang D, Minor M, Chu HW. Electronic cigarette liquid increases inflammation and virus infection in primary human airway epithelial cells. PLOS ONE. 2014;9(9):e108342.

24. LeVine AM, Whitsett JA, Hartshorn KL, Crouch EC, Korfhagen TR. Surfactant protein D enhances clearance of influenza A virus from the lung in vivo. J Immunol. 2001;167(10):5868-5873.

25. McConnell R, et al. Electronic cigarette use and respiratory symptoms in adolescents. Am J Respir Crit Care Med. 2017;195(8):1043-1049.

26. Telenga ED, et al. Untargeted lipidomic analysis in chronic obstructive pulmonary disease. Uncovering sphingolipids. Am J Respir Crit Care Med. 2014;190(2):155-164. 